Material and Methods Retrospective review of our prospectively maintained Neuro IR database and identification of all patients who underwent mechanical thrombectomy for anterior circulation strokes between January 2019 and November 2020. First pass efficacy was defined as successful single-pass mechanical thrombectomy resulting in mTICI score equal or greater than $2 \mathrm{~B}$. Information on number of passes needed to achieve TICI $2 \mathrm{~B}$ or greater as well as total number of passes and final revascularization score were recorded.

Results The Walrus BGC (Q'apel Medical) is a revolutionary 0.087 -inch inner diameter and 0.110 -inch outer diameter device specifically designed for stroke patients. Its design offers distal flexibility, great trackability and optimal proximal support. A total of 239 anterior circulation stroke cases were identified between January 2019 and November 2020. Of those, 80 cases were performed using the Walrus BGC. First pass mTICI score equal or greater than $2 \mathrm{~B}$ was achieved in $45 / 80$ cases $(56.3 \%)$ using the Walrus BGC with 39 of those cases $(86.7 \%)$ achieving mTICI $2 \mathrm{C}$ or 3 . An mTICI score equal or greater than $2 \mathrm{~B}$ was achieved in $77 / 159$ cases $(48.4 \%)$ using other guide catheters including the Flowgate BGC (Stryker), Cello BGC (Medtronic), Merci BGC (Stryker), Neuron Max (Penumbra) and Infinity (Stryker) with 59 of those cases $(76.6 \%)$ achieving mTICI $2 \mathrm{C}$ or 3 . mTICI $2 \mathrm{~B}$ was not achieved in 35 cases $(43.7 \%)$ in the Walrus BGC group versus 82 cases $(51.6 \%)$ in the other guide catheter group. The higher first pass and final recanalization scores maybe explained by the ability to put larger ID intermediate catheters for either aspiration alone or with stent retrievers in conjunction with the Walrus BGC.

Conclusion The Walrus BGC achieved more first pass mTICI scores equal or greater than $2 \mathrm{~B}$ when compared to other guide catheters for anterior circulation mechanical thrombectomy and among those cases also achieved more mTICI 2C and 3 recanalizations.

Disclosures A. Kuhn: None. V. Naragum: None. J. Singh: None. K. de Macedo Rodrigues: None. F. Massari: None. V. Anagnostakou: None. M. Gounis: 1; C; National Institutes of Health (NIH), the United States - Israel Binational Science Foundation, Anaconda, ApicBio, Axovant, Cerenovus, Cook Medical, Gentuity, Imperative Care, InNeuroCo, Magneto. 2; C; Cerenovus, Imperative Care, phenox, Medtronic Neurovascular, Route 92 Medical, Stryker Neurovascular. 4; C; Imperative Care, InNeuroCo and Neurogami. A. Puri: 1; C; NIH, Stryker Neurovascular, Medtronic, Cerenovus. 2; C; Microvention, QApel, Perfuze Medical, Arsenal Medical, Merit Medical, Stryker Neurovascular, Medtronic, Cerenovus. 4; C; InNeuroCo Inc, Galaxy therapeutics, Agile Medical, Perfuze medical and NTI.

\section{E-121 EVALUATION OF SAFETY AND EFFICACY OF SNUFFBOX (DISTAL RADIAL ARTERY) ACCESS FOR MECHANICAL THROMBECTOMY IN ACUTE ISCHEMIC STROKE}

${ }^{1} \mathrm{~A}$ Kuhn, ${ }^{1} \mathrm{~J}$ Singh, ${ }^{1} \mathrm{~F}$ Massari, ${ }^{2} \mathrm{~K}$ de Macedo Rodrigues, ${ }^{1} \mathrm{~V}$ Naragum, ${ }^{1} \mathrm{~V}$ Anagnostakou, ${ }^{1} \mathrm{M}$ Gounis, ${ }^{1} \mathrm{~A}$ Puri*. ${ }^{1}$ Division of Neurointerventional Radiology, Department of Radiology and New England Center for Stroke, University of Massachusetts, Worcester, MA; ${ }^{2}$ Greensboro Radiology, Greensboro, NC

\subsection{6/neurintsurg-2021-SNIS.216}

Purpose To evaluate the safety and efficacy of snuffbox (distal radial artery) access for mechanical thrombectomy in patients presenting with acute ischemic strokes.
Material and Methods High-volume, single center, retrospective analysis of our neurointerventional database between January 2019 and November 2020 and a collection of all patients who underwent mechanical thrombectomy via snuffbox access for treatment of anterior or posterior circulation strokes. Patient demographic information, procedural and outcome data were obtained.

Results A total of 256 anterior $(n=239)$ and posterior $(n=17)$ circulation strokes were identified between January 2019 and November 2020. Of those, 7 were performed via snuffbox access (2.7\%), with 2 strokes located in the anterior and 5 in the posterior circulation. Three patients were female. Mean patient age was 64.1 years (range: 48 to 77 years). The anterior circulation occlusions were located at the M3/MCA and A3/ACA. The posterior circulation occlusions involved the basilar artery. The NIHSS ranged between 2 to 7 (mean NIHSS 5). None of the patients received IV tPA. TICI 3 recanalization was achieved in all cases. Single pass success was seen in 4 cases $(57.1 \%)$. Number of total passes ranged between 1 to 3 . The Benchmark (Penumbra) and Infinity (Stryker Neurovascular) catheters were mainly used as guide catheters for the intervention. Aspiration catheters used included the 5F Sofia distal access catheter (Microvention), Large Bore aspiration catheter (Cerenovus) and Catalyst 6 (Stryker). A stent retriever, either Trevo (Stryker) or Solitaire (Medtronic), was used in all cases. No procedural complications or intracranial hemorrhage was seen. No access site complications were noted. Good clinical outcome with mRS equal or less than 2 was seen in 4 cases $(57.1 \%)$. Two patients died.

Conclusion Snuffbox (distal radial artery) access for mechanical thrombectomy in acute ischemic stroke is feasible and safe. It represents a good alternative to the traditional femoral approach, especially in cases of posterior circulation strokes, patients on anticoagulation or with large body habitus.

Disclosures A. Kuhn: None. J. Singh: None. F. Massari: None. K. de Macedo Rodrigues: None. V. Naragum: None. V. Anagnostakou: None. M. Gounis: 1; C; National Institutes of Health (NIH), the United States - Israel Binational Science Foundation, Anaconda, ApicBio, Axovant, Cerenovus, Cook Medical, Gentuity, Imperative Care, InNeuroCo, Magneto. 2; C; Cerenovus, Imperative Care, phenox, Medtronic Neurovascular, Route 92 Medical, Stryker Neurovascular. 4; C; Imperative Care, InNeuroCo and Neurogami. A. Puri: 1; C; NIH, Stryker Neurovascular, Medtronic, Cerenovus. 2; C; Microvention, QApel, Perfuze Medical, Arsenal Medical, Merit Medical, Stryker Neurovascular, Medtronic, Cerenovus. 4; C; InNeuroCo Inc, Galaxy therapeutics, Agile Medical, Perfuze medical and NTI.

\section{E-122 INCIDENCE OF ACCESS SITE COMPLICATIONS AFTER TRANSRADIAL ACCESS FOR NEUROINTERVENTIONS}

${ }^{1} \mathrm{~A}$ Kuhn, ${ }^{1} \mathrm{~J}$ Singh, ${ }^{1} \mathrm{~A}$ McGowan, ${ }^{1} \mathrm{M}$ Kirk, ${ }^{1} \mathrm{~F}$ Massari, ${ }^{2} \mathrm{~K}$ de Macedo Rodrigues, ${ }^{1} \mathrm{~V}$ Naragum, ${ }^{1} \mathrm{~V}$ Anagnostakou, ${ }^{1} \mathrm{M}$ Gounis, ${ }^{1} \mathrm{~A}$ Puri*. 'Division of Neurointerventional Radiology, Dept of Radiology, New England Center for Stroke Research, University of Massachusetts, Worcester, MA; ${ }^{2}$ Greensboro Radiology, Greensboro, NC

\subsection{6/neurintsurg-2021-SNIS.217}

Purpose To report the incidence of access site complications after transradial (distal radial (anatomical snuffbox) and radial artery) access for diagnostic and interventional Neuro IR procedures. 
Material and Methods We retrospectively reviewed our prospectively maintained Neuro IR database and identified all patients who underwent transradial access for their diagnostic or interventional procedure between May 2019 and January 2021. Patient demographics, clinical information, procedural and radiographic data were collected.

Results We identified 749 patients in whom transradial access was obtained for either diagnostic and interventional Neuro IR procedures. Access was obtained using a 5F/6F/7F radial sheath. In some procedures, the sheath was exchanged for the guide catheter which was then inserted in a bareback (sheathless) fashion. Interventional procedures performed included carotid artery stenting, stroke thrombectomy, intracranial stenting, thrombolysis for central retinal artery occlusion, aneurysm treatment (with coiling or stent-assisted coiling, flow diverters or Woven EndoBridge device placement), vasospasm treatment, arteriovenous malformation and dural arteriovenous fistula embolization, middle meningeal artery embolization, and spinal angiography with embolization. A total of 12 access site complications were recorded (1.6\%) with 4 access site hematomas, 3 inflammatory changes at the puncture site, 2 asymptomatic radial artery occlusions, 2 radial artery injuries (1 self-limiting wire perforation and 1 perforation which was coiled via transfemoral route without any clinical sequela) and 1 retained broken microwire which was successfully removed in a subsequent session. None of the complications resulted in permanent local or neurological deficits.

Conclusion The transradial approach for diagnostic and interventional Neuro IR procedures is a safe vascular access choice.

Disclosures A. Kuhn: None. J. Singh: None. A. McGowan: None. M. Kirk: None. F. Massari: None. K. de Macedo Rodrigues: None. V. Naragum: None. V. Anagnostakou: None. M. Gounis: 1; C; National Institutes of Health (NIH), the United States - Israel Binational Science Foundation, Anaconda, ApicBio, Axovant, Cerenovus, Cook Medical, Gentuity, Imperative Care, InNeuroCo, Magneto. 2; C; Cerenovus, Imperative Care, phenox, Medtronic Neurovascular, Route 92 Medical, Stryker Neurovascular. 4; C; Imperative Care, InNeuroCo and Neurogami. A. Puri: 1; C; NIH, Stryker Neurovascular, Medtronic, Cerenovus. 2; C; Microvention, QApel, Perfuze Medical, Arsenal Medical, Merit Medical, Stryker Neurovascular, Medtronic, Cerenovus. 4; C; InNeuroCo Inc, Galaxy therapeutics, Agile Medical, Perfuze medical and NTI.

\section{E-123 EVALUATION OF SAFETY AND EFFICACY OF TRANSRADIAL ACCESS FOR MECHANICAL THROMBECTOMY INACUTE ISCHEMIC STROKE - A SINGLE CENTER EXPERIENCE}

${ }^{1} \mathrm{~A}$ Kuhn, ${ }^{1} \mathrm{~J}$ Singh, ${ }^{2} \mathrm{~K}$ de Macedo Rodrigues, ${ }^{1} \mathrm{~F}$ Massari, ${ }^{1} \mathrm{~F}$ Massari, ${ }^{1} \mathrm{M}$ Gounis, ${ }^{1} \mathrm{~A}$ Puri ${ }^{*}$. ${ }^{1}$ Division of Neurointerventional Radiology, Department of Radiology and New England Center for Stroke, University of Massachusetts, Worcester, MA; ${ }^{2}$ Greensboro Radiology, Greensboro, NC

\subsection{6/neurintsurg-2021-SNIS.218}

Purpose To evaluate the safety and efficacy of transradial (distal radial (snuffbox) and radial artery) access for mechanical thrombectomy in patients presenting with acute ischemic strokes.

Material and Methods Retrospective review of our prospectively maintained Neuro IR database and identification of all patients who underwent mechanical thrombectomy for anterior and posterior circulation strokes between January 2019 and November 2020. Information on patient characteristics, site of occlusion, catheter set up, TICI recanalization status, and patient outcome at 3 months was collected.

Results A total of 256 anterior $(n=239)$ and posterior $(n=17)$ circulation strokes were identified between January 2019 and November 2020. Of those, 17 were performed via transradial access (6.6\%), with 8 strokes located in the anterior and 9 in the posterior circulation. Eight patients were female. Mean age of anterior circulation stroke patients was 73.9 years (range 46-96 years) and 57.9 years (range 35-76 years) for posterior circulation stroke patients. Most occlusions were seen in the middle cerebral and basilar arteries. The NIHSS ranged between 5 to 24 for anterior circulation strokes (mean NIHSS 13) and 2 to 38 for posterior circulation strokes (mean NIHSS 12). Four patients received IV tPA. Ten procedures were performed via radial artery access and 7 procedures via distal radial artery (snuffbox) access. No conversion to femoral access was needed. Successful recanalization with TICI equal to or higher than $2 \mathrm{~B}$ was achieved in $87.5 \%$ of anterior circulation and $100 \%$ of posterior circulation thrombectomies. TICI $2 \mathrm{C}$ and 3 was achieved in $75 \%$ of anterior circulation and $88.9 \%$ pf posterior circulation thrombectomies. Single pass TICI $2 \mathrm{c}$ and 3 was seen in $7 / 17$ cases $(41.2 \%)$. The Infinity (Stryker) and Benchmark (Penumbra) catheters were mainly used as guide catheters. Intermediate catheters used included the 5F Sofia distal access catheter (Microvention), Large Bore aspiration catheter (Cerenovus) and Catalyst 6 (Stryker). A stent retriever, either Trevo (Stryker) or Solitaire (Medtronic), was used in all cases. No access site complication was noted.

Conclusion Transradial access for mechanical thrombectomy in acute ischemic stroke is a primary access in tortuous vasculature/posterior circulation strokes and is a good alternative to the femoral approach. This vascular access is especially helpful in patients with posterior circulation strokes, on anticoagulation or with large body habitus.

Disclosures A. Kuhn: None. J. Singh: None. K. de Macedo Rodrigues: None. F. Massari: None. F. Massari: None. M. Gounis: 1; C; National Institutes of Health (NIH), the United States - Israel Binational Science Foundation, Anaconda, ApicBio, Axovant, Cerenovus, Cook Medical, Gentuity, Imperative Care, InNeuroCo, Magneto. 2; C; Cerenovus, Imperative Care, phenox, Medtronic Neurovascular, Route 92 Medical, Stryker Neurovascular. 4; C; Imperative Care, InNeuroCo and Neurogami. A. Puri: 1; C; NIH, Stryker Neurovascular, Medtronic, Cerenovus.. 2; C; Microvention, QApel, Perfuze Medical, Arsenal Medical, Merit Medical, Stryker Neurovascular, Medtronic, Cerenovus.. 4; C; InNeuroCo Inc, Galaxy therapeutics, Agile Medical, Perfuze medical and NTI.

\section{E-124 PIPELINE EMBOLIZATION DEVICE WITH SHIELD TECHNOLOGY IN THE TREATMENT OF RECURRENT CEREBRAL ANEURYSMS USING LVIS STENT}

${ }^{1} \mathrm{~K}$ Takayama*, ${ }^{1} \mathrm{~T}$ Wada, ${ }^{1} \mathrm{H}$ Kishida, ${ }^{2} \mathrm{~K}$ Myouchin, ${ }^{3} \mathrm{~K}$ Kichikawa. ${ }^{1}$ Department of Interventional Neuroradiology/Radiology, Kouseikai Takai Hospital, Tenri, Japan; ${ }^{2}$ Department of Radiology, Nara Medical University, Kashihara, Japan; ${ }^{3}$ Department of Radiology, Nara Medical University, Kashihara, Japan

10.1136/neurintsurg-2021-SNIS.219 